\title{
Spontaneous Epidural Hematoma of Cervical Spine
}

\author{
FIROOZ SALEHPOUR, MD, FARHAD MIRZAEI, MD, MOHAMMAD KAZEMZADEH, MD, SEYED \\ AHMAD NASERI ALAVI, MD \\ Department of Neurosurgery, Faculty of Medicine, Tabriz University of Medical Sciences, Tabriz, Iran
}

\begin{abstract}
Spontaneous cervical epidural hematoma is an uncommon cause of acute spinal cord compression. This is a rare idiopathic condition that leads to acute onset of neurologic deficits, which if not recognized early can have catastrophic consequences. Acute cervical epidural hematoma is definitely a condition of neurologic emergency. Although it is a rare condition, it must be considered in nontraumatic patients with sudden onset of neurologic deficits. Patients with spontaneous spinal epidural hematoma typically present with acute onset of severe back pain, and they rapidly develop signs of compression of the spinal cord or cauda equina. Here, we present a case of a 31-year-old man who presented with acute onset of neck pain with radicular component with progressive neurologic deficit. Emergent magnetic resonance imaging revealed cervical extradural hematoma with cord compression that was promptly evacuated. Functional recovery was achieved within 48 hours. The level of preoperative neurologic deficit and its severity, as well as operative interval, are important factors significantly affecting the postoperative outcome.
\end{abstract}

Lumbar Spine

Keywords: spontaneous hematoma, epidural hematoma, cervical hematoma, spinal cord compression

\section{INTRODUCTION}

Spontaneous spinal epidural hematoma (SSEH) is a rare condition requiring early diagnosis and management to achieve recovery of neurologic function. The incidence of SSEH is estimated to be $0.1 \%$ per 100000 individuals. $^{1}$ It is associated with trauma, coagulopathy, arteriovenous malformation, Paget disease, tumor, infection, malignancy, disc herniation, and postoperative complications. ${ }^{2}$

The onset of signs and symptoms of SSEH is usually sudden, and it is rapidly progressive. The initial and classic clinical presentation is acute onset of radiating and severe neck pain, followed by progressive involvement of spinal cord due to the cord compression. ${ }^{3}$ Some patients present with complete sensory and motor deficits below the injury level, whereas some tend to have intact sensory and/or motor function until the last stages. This has prognostic significance because those with some residual function are more likely to show complete recovery. ${ }^{4}$ Magnetic resonance imaging (MRI) is the modality of choice to diagnose the condition within the first 24 hours of onset, in which an SSEH is isointense to the cord on T1-weighted (T1W) images and is usually hyperintense and heterogeneous on T2-weighted (T2W) images. ${ }^{5}$ By 48 hours the hematoma appears hyperintense on both $\mathrm{T} 1 \mathrm{~W}$ and $\mathrm{T} 2 \mathrm{~W}$ sequences. ${ }^{2,5,6}$ The radiologic differential diagnoses include epidural abscess and spinal epidural lymphoma. ${ }^{7}$

The aim of this study is to report a case of acute SSEH without any previous predisposing factors, with the patient presenting with sudden onset of neck pain followed by motor deficits. The patient was successfully treated surgically.

\section{CASE REPORT}

A 31-year-old man presented with acute onset of severe neck pain with progressive quadriparesis of 6 hours' duration. His symptoms initially started as severe pain in the neck radiating to his left upper limb. His past medical history was unremarkable. There was no history of neck trauma, smoking, drinking, or illicit drug use. The patient did not use any anticoagulants or antiplatelets. He did not mention the history of previous neck pain and symptoms of radiculopathy.

On neurologic examination, muscle forces in both upper limbs were $2 / 5$, with significant left-sided grip weakness. Proximal muscle forces in lower limbs were $1 / 5$. Deep tendon reflexes were absent. Plantar reflexes were flexor. There was urinary incontinence, but cranial nerves and cerebellar functions were normal; decreased sensations to pinprick, light 


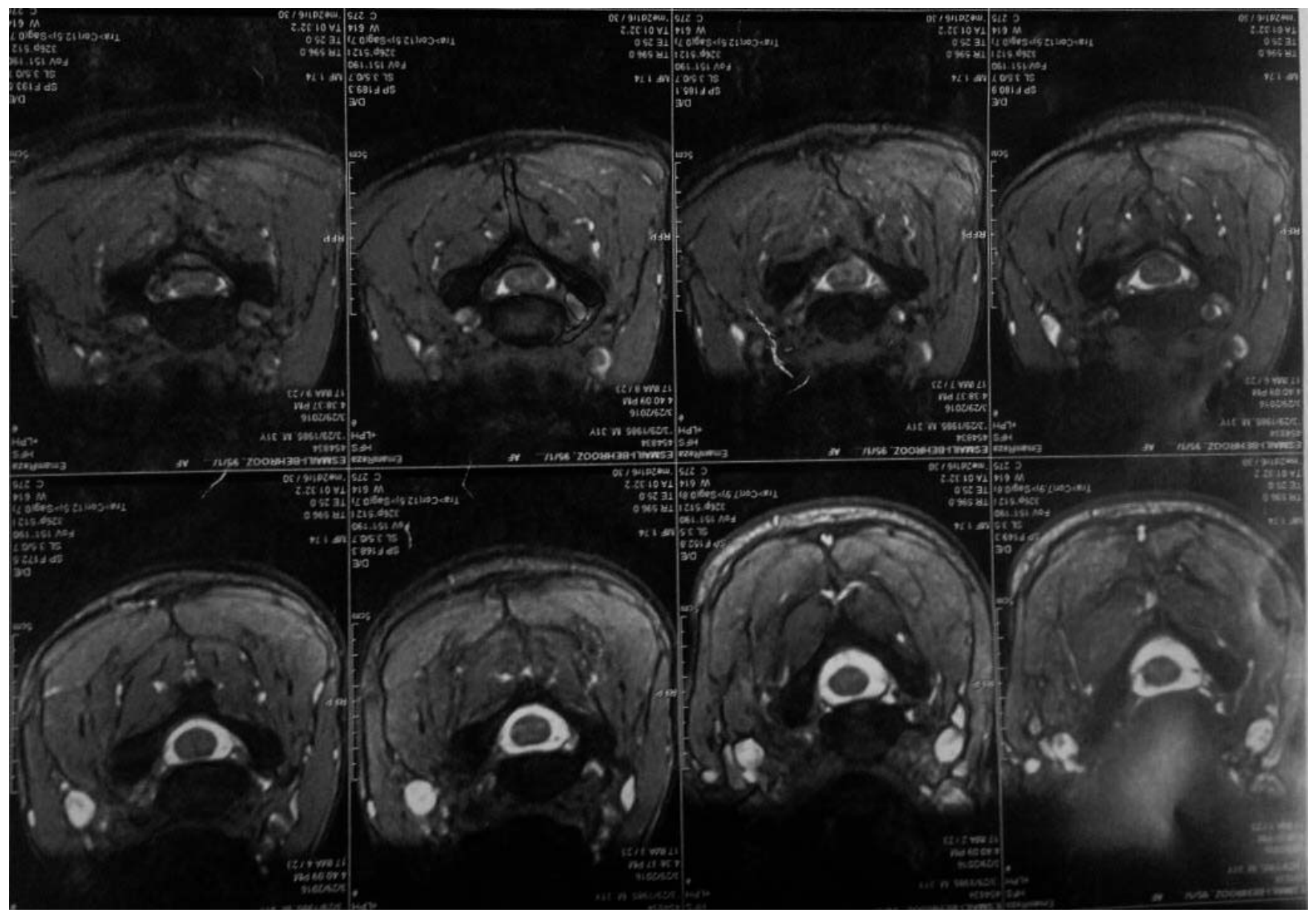

Figure 1. Magnetic resonance imaging showed an isointense to hyperintense lesion mass situated on left side dorsolateral.

touch, and vibration sensations were noted bilaterally. Other physical examinations were normal. His vital signs were normal. Complete blood count was normal. Investigation of coagulation cascade, including international normalized ratio, prothrombin time, activated partial thrombin time, bleeding, and clotting time in the reference lab were all within normal ranges. Furthermore, the patient did not give us any history of abnormal bleeding. Because a cervical spine pathology was suspected, an emergent MRI of the cervical and thoracic spine was performed. The MRI showed an isointense to hyperintense lesion mass situated on the left side dorsolaterally, extending from $\mathrm{C} 5$ to $\mathrm{T} 1$ on $\mathrm{T} 1 \mathrm{~W}$ images, and no enhancement was observed after injection of gadolinium (Figure 1). The T1W images displayed a heterogeneous signal within the lesion (Figures 2 and 3). The final radiologic diagnosis was acute SSEH with significant mass effect and compression of upper cervical cord.

The patient underwent an emergency operation under general anesthesia in a prone position, and after localization of surgery site with a C-arm, C5 to T1 laminectomy was performed. A well-circumscribed clot was seen in the corresponding level. The epidural hematoma was removed easily, and the pressure on the cervical cord was relieved. After the evacuation of solid clots, the dura resumed its

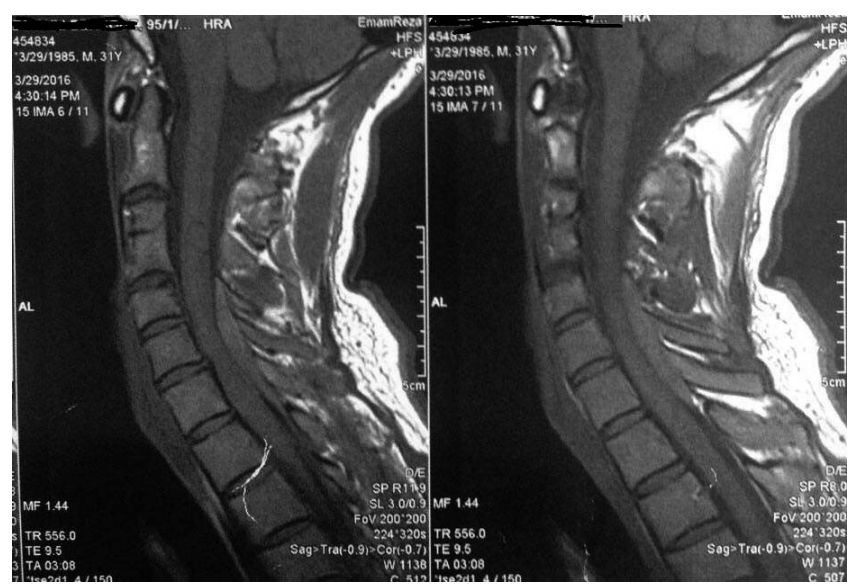

Figure 2. The T1-weighted magnetic resonance imaging showed a heterogeneous signal within the lesion. 


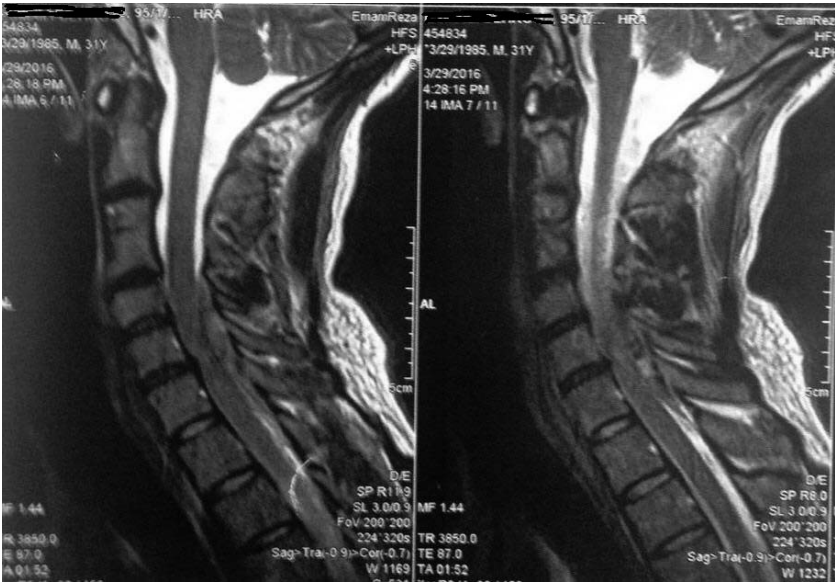

Figure 3. The T2-weighted magnetic resonance imaging showed a heterogeneous signal within the lesion.

normal position and showed good natural pulsation. No other pathology was detected intraoperatively. No discrete bleeding source was identified, and hemostasis was achieved easily. The histopathologic investigation of the specimen was suggestive of an organized hematoma, fibrin deposition, and leukocytes. There was neither evidence of any abnormal vessels nor malignant tissue. After surgery, the patient was admitted to the intensive care unit. Postoperatively the power in all the limbs improved within 24 hours. Motor power in lower limbs improved from $1 / 5$ preoperatively to $4 / 5$, and upper limbs from $2 / 5$ to $4 / 5$ at the ninth day postoperatively. Physical therapy was done during hospitalization. Good recovery of bladder function also was noted. The patient was discharged 10 days after surgery without any complications. He was transferred to the rehabilitation unit for further physical therapies.

\section{DISCUSSION}

A spinal epidural hematoma is a rare spinal pathology which can result in morbidity with delayed treatment or nontreatment. These hematomas are typically considered a surgical emergency. ${ }^{8}$ Spinal epidural hematomas were first described in 1869 and first treated surgically in $1897 .{ }^{9}$ The incidence of this condition is estimated 0 to 1 per 100000 per year. ${ }^{1}$ After the introduction of MRI the incidence and number of diagnosed have further increased. ${ }^{5-7}$ A spinal epidural hematoma can occur in the absence of any trauma or iatrogenic procedures. This definition also includes causes, such as a tumor, coagulopathy, vascular malformation, and cavernous angioma. ${ }^{1,2,4,7}$ The age of patients has bimodal peaks, with an increase in the second and seventh decades. ${ }^{7}$ Halim et al ${ }^{10}$ demonstrated that there is no significant correlation with sex and race. ${ }^{10}$ SSEH can be clinically presented as transverse myelitis, dissecting aortic aneurysm, ruptured cervical herniated disk, epidural neoplasia, or infection, such as an epidural abscess. ${ }^{11}$ The patient's signs and symptoms depend on the location of the epidural hematoma and the degree of cord compression. There is a combination of severe pain and neurologic deficit. Most patients present with severe back or neck pain, often with a radicular component ${ }^{7}$; however, some cases have been reported without neck pain. ${ }^{11}$ After sudden and abrupt pain, motor or sensory deficits occur. The severity and progressiveness of sensory and motor deficit depend on the severity and rapidity of bleeding. ${ }^{7}$ The severity of sensory and motor deficit has a prognostic value; patients with residual function are more likely to show complete recovery than those patients with no motor function. ${ }^{7,8}$ A total of $37 \%$ of cases of SSEH show complete sensory and motor deficit, and the remainder of cases have some sensory or motor function intact. ${ }^{8}$ The clinical picture of SSEH also depends on the location of the hematoma. SSEH may be presented as hemiparesis, hemiplegia, quadriparesis, quadriplegia, and other presentations. The location of SSEH appears to have bimodal peaks at C6 and T12 levels. ${ }^{7,8}$ The extent of involvement is about 2 to 4 (mean, 3.6) vertebral levels in length and is located dorsal to the spinal cord. ${ }^{8}$

The cause of bleeding in the current literature is both venous and arterial in origin. Venous bleeding owing is the commonly accepted hypothesis for the source of the hematoma because spinal epidural veins have no sphincters, and thus have no protection against pressure changing. ${ }^{8}$ This theory seems to be invalid in the cervical region because the venous pressure is low. It is said that the epidural hematoma of the cervical region has an arterial source from free anastomotic arteries in connection with radicular arteries that exist in the epidural space. $^{12}$ The age of a patient with spinal epidural hematoma sets the computed tomography scan appearance. The hematoma has a characteristic convex surface and hyperdense appearance when occurring in the acute stage. ${ }^{11}$ It is difficult to differentiate the hematoma from a tumor, such as a lymphoma or epidural abscess, without administration of contrast medium. ${ }^{2,13}$ MRI is the modality of 
choice to investigate. During the first 24 hours of onset, an epidural hematoma is isointense to the cord on T1W images and is usually hyperintense and heterogeneous on T2W sequences. In first 48 hours, the hematoma appears hyperintense on both $\mathrm{T} 1 \mathrm{~W}$ and T2W images. ${ }^{2,5,6}$ In some studies, several cases have been reported of successfully treated spinal epidural hematomas. All of them presented only with minor neurologic symptoms at the examination and had rapid improvement. ${ }^{1,2,4,8,10,14}$ Although these cases with minimal symptoms and no mass effect on the spinal cord in imaging may be managed successfully with conservative treatment, neurosurgeons should always be aware of the fact that the outcome of SSEH can be very poor when there is a mass effect and/or compression of the spinal cord. ${ }^{8}$

In some cases, during the recovery phase of conservative treatment exacerbation of neurologic deficits may occur, which may require surgical evacuation. Spontaneous recovery in SSEH patients occurs when the hematoma spreads through the spinal epidural spaces enough to decompress the spinal cord. If the patient is a candidate to undergo conservative treatment, close observation with serial neurologic examination and early control MRI are mandatory. ${ }^{8}$ The patients with any neurologic deterioration or onset of new symptoms will undergo surgical intervention. In fact, conservative treatment is reasonable in young patients with minimal neurologic deficits or in patients in whom spontaneous recovery happens. ${ }^{8,12,13}$

In conclusion, it can be concluded that if surgical intervention is the plan of treatment; earlier intervention is better for the outcome. The level of preoperative neurologic deficit and its severity and also operative interval are important factors significantly affecting the postoperative outcome.

\section{REFERENCES}

1. Baek BS, Hur JW, Kwon KY, Lee HK. Spontaneous spinal epidural hematoma. J Korean Neurosurg Soc. 2008;44(1): 40-42.

2. Fukui MB, Swarnkar AS, Williams RL, Acute spontaneous spinal epidural hematomas. AJNR Am J Neuroradiol. 1999;20:1365-1372.

3. Groen R, Ponssen H. The spontaneous spinal epidural hematoma: a study of the etiology. J Neurol Sci. 1990;98:121138.

4. Lan T, Chen Y, Yang XJ, et al. Spontaneous spinal epidural haematoma. J Ortho Trans. 2015;3(3):152-156.

5. Vazquez-Barquero A, Abascal F, Garcia-Valtuille R, Pinto JI, Figols FJ, Cerezal L. Chronic nontraumatic spinal epidural hematoma of the lumbar spine: MRI diagnosis. Eur Radiol. 2000;10(10):1602-1605.

6. Avrahami E, Tadmor R, Ram Z, Feibel M, Itzhak Y. MR demonstration of spontaneous acute epidural hematoma of the thoracic spine. Neuroradiology. 1989;31(1):89-92.

7. Gala FB, Aswani Y. Imaging in spinal posterior epidural space lesions: a pictorial essay. Indian $J$ Radiol Imaging. 2016;26(3):299-315.

8. Gopalkrishnan CV, Dhakoji A, Nair S. Spontaneous cervical epidural hematoma of idiopathic etiology: case report and review of literature. J Spinal Cord Med. 2012;35(2):113117.

9. Jackson R. Case of spinal apoplexy. Lancet. 1869;2(2392):5-6.

10. Halim TA, Nigam V, Tandon V, Chhabra HS. Spontaneous cervical epidural hematoma: report of case managed conservatively. Indian J Orthop. 2008;42(3):357-359.

11. Lee SC, Lui TG, Lee ST. Spontaneous cervical spinal epidural hematoma: a case report. Acta Neurol Taiwan. 2003;12(1):34-37.

12. Beatty RM, Winston KR. Spontaneous cervical epidural hematoma. J Neurosurg. 1984;61(1):143-148.

13. Henderson RD, Pittock SJ, Piepgras DG, Wijdicks EF. Acute spontaneous spinal epidural hematoma. Arch Neurol. 2001;58(7):1145-1146.

14. Holtås S, Heiling M, Lönntoft M. Spontaneous spinal epidural hematoma: findings at MR imaging and clinical correlation. Radiology. 1996;199(2):409-413.

Disclosures and COl: No authors have conflicts of interest associated with this study.

Corresponding Author: Seyed Ahmad Naseri Alavi, Department of Neurosurgery, Faculty of Medicine, Tabriz University of Medical Sciences, Tabriz, Iran. Email: dr.arsalan2010@gmail.com.

Published 30 March 2018

This manuscript is generously published free of charge by ISASS, the International Society for the Advancement of Spine Surgery. Copyright (C) 2018 ISASS. To see more or order reprints or permissions, see http://ijssurgery.com. 